\title{
An example in Beurling's theory of primes
}

\author{
by
}

\section{Eugenio P. Balanzario (Morelia)}

1. Introduction. Let $P=\left\{p_{1}, p_{2}, \ldots\right\}$ be a set of numbers satisfying the following three conditions:

$$
1<p_{1}, \quad p_{k} \leq p_{k+1}, \quad \lim _{k \rightarrow \infty} p_{k}=\infty .
$$

Let $N$ be the set of numbers which are finite products of elements of $P$. Following A. Beurling [1], we call $P$ a set of generalized primes (g-primes) and $N$ a set of generalized integers (g-integers). We denote by $P(x)$ and $N(x)$ the counting functions of the sets $P$ and $N$. Two interesting problems arise. First, we have the Inverse Problem: given the knowledge of the distribution of the set $N$ of g-integers, obtain information about the distribution of the set $P$ of g-primes. The classical Prime Number Theorem is an example of an inverse problem. See also the above cited reference to Beurling [1], as well as H. G. Diamond [2], where the Prime Number Theorem is proved in the setting of the Theory of Generalized Numbers. We also have the Direct Problem: given the knowledge of the distribution of the elements in $P$, obtain information about the distribution of those of $N$. The Direct Problem has been studied by (among others) P. Malliavin [4] and H. G. Diamond [3]. We now summarize their results.

Assume that the distribution of the primes is given by

$$
P(x)=\operatorname{Li}(x)+O\left(x e^{-\sqrt{\log x}}\right) .
$$

We are interested in estimating $N(x)$. P. Malliavin [4] proved that

$$
N(x)=c x+O\left(x \exp \left\{-\theta(\log x)^{0.2}\right\}\right)
$$

for some positive constants $c$ and $\theta$. If we let

$$
A=\text { l.u.b. }\left\{a: N(x)=c x+O\left(x \exp \left\{-\theta(\log x)^{a}\right\}\right)\right\},
$$

1991 Mathematics Subject Classification: Primary 11N80.

This article, with minor changes, is part of the author's Ph.D. dissertation, written at the University of Illinois, Urbana-Champaign, under the direction of Prof. H. G. Diamond. 
then the following estimates are true:

$$
\begin{array}{ll}
A \geq 0.2 & \text { (Malliavin, 1961) } \\
A \geq 0.333 \ldots & \text { (Diamond, 1970). }
\end{array}
$$

In this article we construct a generalized number system to prove that $A \leq 0.5$. That is, we prove the following

THEOREM. There exists a continuous distribution $P(x)$ of prime numbers for which (1) holds and furthermore

$$
N(x)=c x+\Omega\left(x e^{-\theta \sqrt{\log x}}\right)
$$

for some positive constants $c$ and $\theta$.

The words "continuous distribution" in the theorem deserve an explanation. They are to mean that there exists a measure $d P$ (see (4) below) whose integral $\int_{1}^{x} d P$ is a continuous function in the interval $(1, \infty)$. We use this measure as input in the exponential formula

$$
d N=e^{d P}=\delta+d P+\frac{d P * d P}{2 !}+\frac{(d P)^{* 3}}{3 !}+\ldots,
$$

where $\delta$ is the Dirac measure placed at the point 1 and $*$ is the multiplicative (Dirichlet) convolution for measures; the measure $d P * d P$ assigns to each Borel set $E$ the numerical value

$$
\iint_{s t \in E} d P(s) \cdot d P(t)
$$

Moreover, the "set of integers" $N$ is given by $N(x)=\int_{1}^{x} e^{d P}$. We will also make use of the zeta function of this "set of integers" as given by

$$
\zeta(s)=\int_{1}^{\infty} x^{-s} d N(x)=\int_{1}^{\infty} x^{-s} e^{d P}=\exp \left\{\int_{1}^{\infty} x^{-s} d P(x)\right\} .
$$

Finally, we point out that the exponential formula (3) gives the counting measure for the set of ordinary integers when we take $d P$ to be the measure $d \Pi$ where

$$
\Pi(x)=\pi(x)+\frac{1}{2} \pi\left(x^{1 / 2}\right)+\frac{1}{3} \pi\left(x^{1 / 3}\right)+\ldots,
$$

and $\pi(x)$ is the number of ordinary primes not greater than $x$. For a more detailed treatment of these notions see Diamond [3].

2. The example. We start by letting

$$
P(x)=\int_{1}^{x} \frac{1-t^{-k}}{\log t} \gamma(t) d t,
$$


where

$$
\gamma(t)=1-\sum_{n>n_{0}} \alpha_{n} \frac{\cos \left(b_{n} \log t\right)}{t^{a_{n}}}, \quad t \geq 1
$$

We think of $k, n_{0},\left\{\alpha_{n}\right\},\left\{a_{n}\right\}$ and $\left\{b_{n}\right\}$ as parameters to be chosen. The following constants serve our purposes (however, we will carry out some of our calculations for an arbitrary choice of these parameters):

$$
\begin{array}{rlrl}
k & =4, & n_{0} & =3, \\
b_{n} & =\exp \left\{\left(\log x_{n}\right)^{1 / 2}\right\}, & a_{n} & =1 / \log b_{n}=1 / \sqrt{\log x_{n}}, \\
x_{1} & =e^{10}, \\
x_{n+1} & =\exp \left\{\left(\log x_{n}\right)^{2}\right\}, \\
T_{n} & =\exp \left\{\left(\log x_{n}\right)^{3 / 4}\right\}, & \alpha_{n} & =2 / n^{2} .
\end{array}
$$

We also define $\alpha=\sum_{n>n_{0}} \alpha_{n}$, and for $n>n_{0}$ we let $\alpha_{-n}=\alpha_{n}, a_{-n}=a_{n}$ and $b_{-n}=-b_{n}$. We make constant use of this notation.

In order that we may consider $P(x)$ to be a counting function, it is necessary that it be non-decreasing. This is a consequence of the following easy lemma.

LEMMA 1. With $\gamma$ as in (5), we have $\gamma(t) \geq 0$.

3. Estimation of $P(x)$. Now we show that (1) holds. In fact we have a slightly better estimate for $P(x)$.

Proposition 2. If $P(x)$ is given by (4) and $k>1$, then

$$
P(x)=\operatorname{Li}(x)+O\left(x e^{-2 \sqrt{\log x}}\right) .
$$

Proof. We have

$$
\begin{aligned}
P(x) & =\int_{1}^{x} \frac{1-t^{-k}}{\log t}\left(1-\sum_{n>n_{0}} \alpha_{n} \frac{\cos \left(b_{n} \log t\right)}{t^{a_{n}}}\right) d t \\
& =\int_{e}^{x} \frac{1-t^{-k}}{\log t} d t-\sum_{n>n_{0}} \alpha_{n} \int_{e}^{x} \frac{1-t^{-k}}{\log t} \cdot \frac{\cos \left(b_{n} \log t\right)}{t^{a_{n}}} d t+O(1) \\
& =\int_{e}^{x} \frac{d t}{\log t}-\sum_{n>n_{0}} \alpha_{n} \int_{e}^{x} \frac{\cos \left(b_{n} \log t\right)}{t^{a_{n}} \log t} d t+O(1),
\end{aligned}
$$

because $k>1$. Now we show that

$$
\sum_{n>n_{0}} \alpha_{n} \int_{e}^{x} \frac{\cos \left(b_{n} \log t\right)}{t^{a_{n}} \log t} d t=O\left(x e^{-2 \sqrt{\log x}}\right) .
$$


To this end, notice that

$$
\begin{aligned}
\left|\int_{e}^{x} \frac{\cos \left(b_{n} \log t\right)}{t^{a_{n}} \log t} d t\right|= & \left|\int_{1}^{\log x} \frac{\cos \left(b_{n} t\right)}{t} e^{t\left(1-a_{n}\right)} d t\right| \\
= & \mid\left[\frac{\sin \left(b_{n} t\right)}{b_{n} t} e^{t\left(1-a_{n}\right)}\right]_{1}^{\log x} \\
& -\frac{1}{b_{n}} \int_{1}^{\log x} \sin \left(b_{n} t\right) \frac{e^{t\left(1-a_{n}\right)}}{t}\left(1-a_{n}-\frac{1}{t}\right) d t \mid \\
\leq & 2 \frac{x^{1-a_{n}}}{b_{n} \log x}+\frac{1}{b_{n}} \int_{1}^{\log x} \frac{e^{t\left(1-a_{n}\right)}}{t} d t .
\end{aligned}
$$

To estimate the last integral we notice that $e^{t\left(1-a_{n}\right)} / t$ reaches a minimum value at $t=1 /\left(1-a_{n}\right) \rightarrow 1$ as $n \rightarrow \infty$. After attaining its minimum the integrand increases to infinity, thus, the largest value of the integrand is achieved when $t=\log x$. Hence

$$
\left|\int_{e}^{x} \frac{\cos \left(b_{n} \log t\right)}{t^{a_{n}} \log t} d t\right| \leq 2 \frac{x^{1-a_{n}}}{b_{n} \log x}+\frac{1}{b_{n}} \cdot \frac{x^{1-a_{n}}}{\log x} \log x \leq 3 \frac{x^{1-a_{n}}}{b_{n}} .
$$

By the definition of $a_{n}$ and $b_{n}$ we have

$$
\frac{x^{1-a_{n}}}{b_{n}}=x \exp \left\{-\frac{\log x}{\sqrt{\log x_{n}}}-\sqrt{\log x_{n}}\right\} .
$$

Let $A^{2}=\log x$ and $B^{2}=\log x_{n}$. From $(A-B)^{2} \geq 0$, we deduce that $-A^{2} / B-B \leq-2 A$. Hence

$$
\frac{x^{1-a_{n}}}{b_{n}}=x \exp \left\{-\frac{A^{2}}{B}-B\right\} \leq x e^{-2 A}=x e^{-2 \sqrt{\log x}} .
$$

Therefore

$$
\sum_{n>n_{0}} \alpha_{n}\left|\int_{e}^{x} \frac{\cos \left(b_{n} \log t\right)}{t^{a_{n}} \log t} d t\right| \leq \sum_{n>n_{0}} 3 \alpha_{n} x e^{-2 \sqrt{\log x}}=3 \alpha x e^{-2 \sqrt{\log x}} .
$$

This proves Proposition 2.

4. Estimation of $N(x)$. We now define our zeta function for $s=\sigma+i t$ with $\sigma>1$ as

$$
\zeta(s)=\int_{1}^{\infty} x^{-s} d N(x)=\exp \left\{\int_{1}^{\infty} x^{-s} d P(x)\right\} .
$$


By inverting the first equation in (6) we get

$$
N(x)=\frac{1}{2 \pi i} \int_{b-i \infty}^{b+i \infty} \zeta(s) \frac{x^{s}}{s} d s, \quad b>1 .
$$

Furthermore, if

$$
M(x)=\int_{1}^{x} N(t) d t
$$

then

$$
M(x)=\frac{1}{2 \pi i} \int_{b-i \infty}^{b+i \infty} \zeta(s) \frac{x^{s+1}}{s(s+1)} d s, \quad b>1 .
$$

To prove that (2) is true it suffices to show that for some $\theta^{*}<\theta$,

$$
M(x)=\frac{c}{2} x^{2}+\Omega\left(x^{2} e^{-\theta^{*} \sqrt{\log x}}\right) .
$$

Indeed, if (2) is not true then

$$
N(x)=c x+o\left(x e^{-\theta \sqrt{\log x}}\right),
$$

so that

$$
M(x)=\int_{1}^{x}\left\{c t+o\left(t e^{-\theta \sqrt{\log t}}\right)\right\} d t=\frac{c}{2} x^{2}+o\left(x^{2} e^{-\theta^{*} \sqrt{\log x}}\right) .
$$

This is a contradiction.

We will prove that (8) is true for $\theta^{*}=3+\varepsilon$ and hence that (2) holds for $\theta>3$.

The next proposition is important for our purposes because it expresses our zeta function as defined in (6) as an infinite product, from which we can read off its singularities. As a matter of fact, we have chosen the parameters $\left\{a_{n}\right\}$ and $\left\{b_{n}\right\}$ in such a way as to give us a distribution of the singularities of $\zeta(s)$ in the complex plane, from which we can deduce the oscillation statement (8). In order to state it recall that for $n>n_{0}$, we have $a_{-n}=a_{n}$, $b_{-n}=-b_{n}$ and $\alpha_{-n}=\alpha_{n}$.

Proposition 3. Consider the zeta function

$$
\zeta(s)=\exp \left\{\int_{1}^{\infty} x^{-s} d P(x)\right\},
$$

where $d P$ is given by (4) above. Then, for $\Re(s)>1$,

$$
\zeta(s)=\frac{s+k-1}{s-1} \prod_{|n|>n_{0}}\left(1-\frac{k}{s-1+a_{n}-i b_{n}+k}\right)^{\alpha_{n} / 2} .
$$

Lemma 4. If we define $\gamma(t)$ by (5) and

$$
\gamma_{N}(t)=1-\sum_{n_{0}<n \leq N} \alpha_{n} \frac{\cos \left(b_{n} \log t\right)}{t^{a_{n}}}, \quad t \geq 1
$$


then $\gamma_{N}(t)$ converges uniformly to $\gamma(t)$ for $t \geq 1$ and in fact $\left|\gamma(t)-\gamma_{N}(t)\right|$ $\leq 2 / N$.

Pr o of (of Proposition 3). Notice first that

$$
\frac{\cos (b \log t)}{t^{a}}=\frac{1}{2 t^{a}}\left(e^{i b \log t}+e^{-i b \log t}\right)=\frac{1}{2}\left(t^{-a+i b}+t^{-a-i b}\right) .
$$

Hence

$$
\frac{\cos (b \log t)}{t^{a}} \cdot \frac{1-t^{-k}}{\log t} d t=\frac{1}{2}\left(t^{-a-i b}+t^{-a+i b}\right) \frac{1-t^{-k}}{\log t} d t .
$$

Thus, for $\Re(s)>1$, we have

$$
\begin{aligned}
& -\frac{d}{d s} \int_{1}^{\infty} t^{-s} \frac{\cos (b \log t)}{t^{a}} \cdot \frac{1-t^{-k}}{\log t} d t \\
& \quad=\frac{1}{2} \int_{1}^{\infty}\left(t^{-s-a-i b}+t^{-s-a+i b}-t^{-s-a-i b-k}-t^{-s-a+i b-k}\right) d t \\
& \quad=\frac{d}{d s} \log \left[\left(1-\frac{k}{s-1+a+i b+k}\right)^{1 / 2}\left(1-\frac{k}{s-1+a-i b+k}\right)^{1 / 2}\right] .
\end{aligned}
$$

Therefore, we have

$$
\begin{aligned}
& -\int_{1}^{\infty} t^{-s} \frac{\cos (b \log t)}{t^{a}} \cdot \frac{1-t^{-k}}{\log t} d t \\
& =\log \left\{\left(1-\frac{k}{s-1+a+i b+k}\right)^{1 / 2}\left(1-\frac{k}{s-1+a-i b+k}\right)^{1 / 2}\right\}+\text { Const. }
\end{aligned}
$$

By taking the limit as $\Re(s)$ tends to infinity we see that the constant of integration is zero.

Similarly, we have

$$
\int_{1}^{\infty} t^{-s} \frac{1-t^{-k}}{\log t} d t=\log \frac{s+k-1}{s-1} .
$$

Thus, from the definition of $\gamma_{N}$, we get

$$
\begin{aligned}
& \int_{1}^{\infty} t^{-s} \frac{1-t^{-k}}{\log t} \gamma_{N}(t) d t \\
&=\log \left(\frac{s+k-1}{s-1}\right)+\sum_{n_{0}<|n| \leq N} \alpha_{n} \log \left(1-\frac{k}{s-1+a_{n}-i b_{n}+k}\right)^{1 / 2} \\
&=\log \left\{\frac{s+k-1}{s-1} \prod_{n_{0}<|n| \leq N}\left(1-\frac{k}{s-1+a_{n}-i b_{n}+k}\right)^{\alpha_{n} / 2}\right\} .
\end{aligned}
$$

Taking the limit as $N \rightarrow \infty$ yields the assertion. 
The infinite product representation of $\zeta(s)$ given in (9) holds not only in the half plane $\Re(s)>1$, but in a larger region. To show this, we prove the following proposition, which will also give us a useful upper bound for $|\zeta(s)|$ in the extended domain of definition.

Proposition 5. If $s=\sigma+i t$ is such that $\sigma>-k+2, \alpha=\sum_{n>n_{0}} \alpha_{n}$, and if

$$
\varphi(s)=\prod_{|n|>n_{0}}\left(1-\frac{k}{s-1+a_{n}-i b_{n}+k}\right)^{\alpha_{n} / 2}
$$

then

$$
|\varphi(s)| \leq(k+1) e^{\alpha} .
$$

Proof. Let $s=\sigma+i t$ be given. Since $b_{n}=\exp \left\{\sqrt{\log x_{n}}\right\}$ we have

$$
\begin{aligned}
b_{n+1}-b_{n} & =\exp \left\{\sqrt{\log x_{n+1}}\right\}-\exp \left\{\sqrt{\log x_{n}}\right\} \\
& =\exp \left\{\log x_{n}\right\}-\exp \left\{\sqrt{\log x_{n}}\right\} \geq x_{n}-\sqrt{x_{n}} \geq 4 k
\end{aligned}
$$

for all $n$, since $x_{n} \geq x_{1}=e^{10}$ and $k<5000$. Therefore the interval $(t-2 k, t+2 k)$ contains at most one element of $\left\{b_{n}\right\}$. Denote this element (if it exists!) by $b_{n(t)}$. We now write

$$
=\left|1-\frac{k}{s-1+a_{n(t)}-i b_{n(t)}+k}\right|^{\alpha_{n(t)} / 2} \prod_{\substack{|n|>n_{0} \\ n \neq n(t)}}\left|1-\frac{k}{s-1+a_{n}-i b_{n}+k}\right|^{\alpha_{n} / 2} .
$$

Since $a_{n}>0$, we have $\sigma-1+a_{n}+k>\sigma-1+k>1$ and hence

$$
\left|1-\frac{k}{s-1+a_{n(t)}-i b_{n(t)}+k}\right|^{\alpha_{n(t)} / 2} \leq\left|1+\frac{k}{\sigma-1+k}\right|^{\alpha_{n(t)} / 2} \leq 1+k .
$$

On the other hand, when $n \neq n(t)$,

$$
\begin{aligned}
\left|1-\frac{k}{s-1+a_{n}-i b_{n}+k}\right|^{\alpha_{n} / 2} & =\exp \left\{\frac{\alpha_{n}}{2} \log \left|1-\frac{k}{s-1+a_{n}-i b_{n}+k}\right|\right\} \\
& =\exp \left\{\frac{\alpha_{n}}{2} \Re \log \left(1-\frac{k}{s-1+a_{n}-i b_{n}+k}\right)\right\} \\
& =\exp \left\{\frac{\alpha_{n}}{2} \Re\left(-z-\frac{z^{2}}{2}-\frac{z^{3}}{3}-\ldots\right)\right\}
\end{aligned}
$$


where

$$
|z|=\left|\frac{k}{s-1+a_{n}-i b_{n}+k}\right| \leq \frac{k}{\left|\Im(s)-b_{n}\right|}=\frac{k}{\left|t-b_{n}\right|} \leq \frac{k}{2 k}=\frac{1}{2} .
$$

Therefore

$$
\begin{aligned}
|\varphi(s)| & \leq(k+1) \prod_{\substack{|n|>n_{0} \\
n \neq n(t)}} \exp \left\{\frac{\alpha_{n}}{2}\left(|z|+\frac{|z|^{2}}{2}+\frac{|z|^{3}}{3}+\ldots\right)\right\} \\
& \leq(k+1) \exp \left\{\frac{1}{4} \sum_{|n|>n_{0}} \alpha_{n}\left(1+\frac{1}{2}+\frac{1}{4}+\ldots\right)\right\} \\
& =(k+1) \exp \left\{\frac{1}{2} \sum_{|n|>n_{0}} \alpha_{n}\right\}=(k+1) e^{\alpha}
\end{aligned}
$$

as claimed.

For the particular case $k=4, n_{0}=3$, and $\alpha_{n}=2 n^{-2}$ we have

$$
|\varphi(s)| \leq 5 \exp \left\{2 \sum_{n>3} \frac{1}{n^{2}}\right\}<9 \quad \text { if } \sigma>-2 .
$$

Let $D_{\zeta}$ be the region defined by

$$
\begin{aligned}
& D_{\zeta}=\{s=\sigma+i t \in \mathbb{C}: \sigma>-k+2, \\
& s \neq \xi\left(1-a_{n}+i b_{n}\right)+(1-\xi)\left(1-a_{n}+i b_{n}-k\right) \\
&\text { for any } \left.0 \leq \xi \leq 1,|n| \geq n_{0}\right\} .
\end{aligned}
$$

By a theorem of Weierstrass on the uniform convergence of analytic functions, we know that $\varphi(s)$ is analytic on $D_{\zeta}$. The equation

$$
\zeta(s)=\frac{s+k-1}{s-1} \varphi(s), \quad \sigma>1,
$$

gives us an analytic continuation of $\zeta(s)$ to $D_{\zeta}$ with $s=1$ removed, where $\zeta(s)$ has a simple pole. Notice that, since the zeros of $\varphi(s)$ are of fractional order, we avoid problems of multiple-valuedness by restricting the domain of definition of $\zeta(s)$ to $D_{\zeta}$.

Corollary 6. For $s \in D_{\zeta}$ such that $|s-1|>1$ we have $|\zeta(s)| \leq 45$.

Pro of. We have

$$
|\zeta(s)|=\left|\frac{s+k-1}{s-1} \varphi(s)\right| \leq 9\left|1+\frac{k}{s-1}\right| \leq 9\left(1+\frac{4}{|s-1|}\right) .
$$

Our next step is to estimate $M(x)$ (cf. (7)) where

$$
x=x_{n}\left(1+\frac{\theta_{1}}{\log x_{n}}\right), \quad\left|\theta_{1}\right|<1 .
$$


REMARK. It would be more accurate to write $w_{n}$, for example, in place of $x$. We prefer to write $x$ in order to keep our formulas simple. We will choose $\theta_{1}$ (and hence $x$ ) in such a way that $M(x)$ equals the main term $c x$, plus a large "error term".

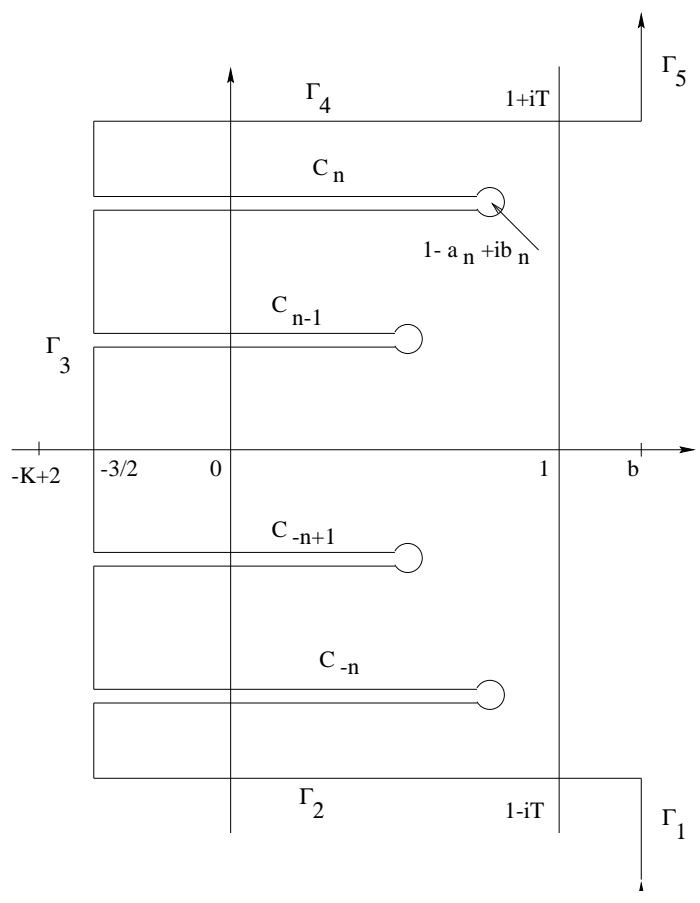

Fig. 1

We deform the vertical path of integration in the inversion formula (7) from the path $\Re(s)=b>1$ to a path $\Gamma_{1} \cup \Gamma_{2} \cup \Gamma_{3}^{*} \cup \Gamma_{4} \cup \Gamma_{5} \subset D_{\zeta}$ (see Figure 1$)$. Here $\Gamma_{1}$ joins $b-i \infty$ to $b-i T_{n}$, where $T_{n}=\exp \left\{\left(\log x_{n}\right)^{3 / 4}\right\}$. The points $b-i T_{n}$ to $-3 / 2+i T_{n}$ are joined by $\Gamma_{2}$. The segments $\Gamma_{5}$ and $\Gamma_{4}$ are symmetric to $\Gamma_{1}$ and $\Gamma_{2}$ with respect to the horizontal axis. We denote by $\Gamma_{3}^{*}$ a comb formed by horizontal loops $C_{m}, n_{0}<|m| \leq n$, each going around the singular point $1-a_{m}+i b_{m}$. The collection of vertical line segments joining one loop to the next one is denoted by $\Gamma_{3}$. The points on $\Gamma_{3}$ have real part equal to $-3 / 2$. Furthermore, each $C_{m}$ is made up of two horizontal line segments joined at the right hand side by a small circle with center at $1-a_{m}+i b_{m}$. The two horizontal line segments of $C_{m}$ are extended to the left until they meet $\Gamma_{3}$.

Now we write

$$
M(x)=I_{1}+\ldots+I_{5}+J_{-n}+\ldots+J_{+n}+\{\text { residues at } s=0,1\}
$$


where

$$
\begin{array}{rlrl}
I_{m} & =\frac{1}{2 \pi i} \int_{\Gamma_{m}} \zeta(s) \frac{x^{s+1}}{s(s+1)} d s, & & m=1, \ldots, 5, \\
J_{m} & =\frac{1}{2 \pi i} \int_{C_{m}} \zeta(s) \frac{x^{s+1}}{s(s+1)} d s, & n_{0}<|m| \leq n .
\end{array}
$$

Here, as above, $C_{m}$ is the $m$ th horizontal loop with imaginary part equal to $b_{m}$.

Consider first the integral $I_{3}$. As a matter of fact, in this case we do not have just one integral but many of them. This is because the vertical segment $\Gamma_{3}$ is broken at each horizontal loop $C_{m}$. However, on each vertical component of $\Gamma_{3}$ the integrand is bounded by the same constant: 45 . Thus, since $\Re(s)=-3 / 2$ on $\Gamma_{3}$, we have

$$
\begin{aligned}
\left|I_{3}\right| & \leq \frac{1}{2 \pi} \int_{-\infty}^{\infty} 45 \frac{x^{-3 / 2+1}}{|(-3 / 2+i t)(1 / 2+i t)|} d t \leq \frac{8}{\sqrt{x}} \int_{0}^{\infty} \frac{d t}{1 / 4+t^{2}} \\
& \leq \frac{8}{\sqrt{x}}\left\{\int_{0}^{1 / 2} 4 d t+\int_{1 / 2}^{\infty} \frac{d t}{t^{2}}\right\}=\frac{8}{\sqrt{x}}\left(2+\frac{1}{1 / 2}\right)=\frac{32}{\sqrt{x}} .
\end{aligned}
$$

Let $b=1+1 / \log x_{n}$. Then $\left|I_{2}\right|$ and $\left|I_{4}\right|$ are both less than $58\left(x / T_{n}\right)^{2}$. Indeed, each is at most

$$
\begin{aligned}
\frac{1}{2 \pi} \int_{-3 / 2}^{1+\left(\log x_{n}\right)^{-1}} 45 & \frac{x^{2+\left(\log x_{n}\right)^{-1}}}{T_{n}^{2}} d \sigma \\
& \leq \frac{8}{T_{n}^{2}} x^{2+\left(\log x_{n}\right)^{-1}} \int_{-3 / 2}^{1+1 / 10} d \sigma \\
& \leq 21\left(\frac{x}{T_{n}}\right)^{2} \exp \left\{\frac{1}{\log x_{n}} \log \left(x_{n}\left(1+\frac{\theta_{1}}{\log x_{n}}\right)\right)\right\} \\
& \leq 21\left(\frac{x}{T_{n}}\right)^{2} \exp \left\{1+\left(\frac{1}{\log x_{n}}\right)^{2}\right\} \leq 58\left(\frac{x}{T_{n}}\right)^{2} .
\end{aligned}
$$

Now we consider the integrals $I_{1}$ and $I_{5}$ : each of $\left|I_{1}\right|$ and $\left|I_{5}\right|$ is at most

$$
\begin{aligned}
\frac{1}{2 \pi} \int_{T_{n}}^{\infty} 45 \frac{x^{2+\left(\log x_{n}\right)^{-1}}}{t^{2}} d t & \leq 8 x^{2} \exp \left\{1+\left(\frac{1}{\log x_{n}}\right)^{2}\right\} \frac{1}{T_{n}} \\
& \leq 8 \frac{x^{2}}{T_{n}} e^{1.01} \leq 22 \frac{x^{2}}{T_{n}} .
\end{aligned}
$$


From the above estimates we get

$$
M(x)=k \varphi(1) \frac{1}{2} x^{2}+(1-k) \varphi(0) x+\sum_{m=-n}^{n} J_{m}+O\left(x^{2} / T_{n}\right),
$$

where the $O$-constant is less than $2(58+22)+32=192$ (because $1 / \sqrt{x} \leq$ $\left.x^{2} / T_{n}\right)$. Let us examine the expression

$$
x^{2} / T_{n}=x^{2} \exp \left\{-\left(\log x_{n}\right)^{3 / 4}\right\} .
$$

We want to write $x$ in place of $x_{n}$ in the above expression. To do this notice first that, since $x_{1} \geq e^{10}$, we have $x_{n+1}=x_{n}^{\log x_{n}} \geq x_{n}^{10}$ so that

$$
x_{n} \geq x_{n-1}^{10} \geq x_{n-2}^{10^{2}} \geq \ldots \geq x_{n-(n-1)}^{10^{n-1}} \geq e^{10^{n}} .
$$

Thus we get $\log x_{n} \geq 10^{n}$ and hence $n \leq \log \log x_{n}$.

Lemma 7. If $x_{n}$ is as in (10) then $\left|\log x-\log x_{n}\right| \leq 2 / 10^{n}$.

Proof. We have

$$
\begin{aligned}
\log x & =\log \left[x_{n}\left(1+\frac{\theta_{1}}{\log x_{n}}\right)\right], \quad\left|\theta_{1}\right|<1, \\
& =\log x_{n}+\log \left(1+\frac{\theta_{1}}{\log x_{n}}\right) .
\end{aligned}
$$

Thus

$$
\begin{aligned}
\left|\log x-\log x_{n}\right| & \leq \frac{\left|\theta_{1}\right|}{\log x_{n}}\left(1+\frac{\left|\theta_{1}\right|}{\log x_{n}}+\left(\frac{\left|\theta_{1}\right|}{\log x_{n}}\right)^{2}+\ldots\right) \\
& \leq \frac{1}{\log x_{n}}\left(1+\frac{1}{10}+\left(\frac{1}{10}\right)^{2}+\ldots\right) \leq \frac{2}{\log x_{n}} \leq \frac{2}{10^{n}} .
\end{aligned}
$$

By the mean value theorem of differential calculus, there is a number $\xi_{n}$ between $x$ and $x_{n}$ such that

$$
\left|(\log x)^{3 / 4}-\left(\log x_{n}\right)^{3 / 4}\right|=\frac{3}{4}\left(\frac{1}{\log \xi_{n}}\right)^{1 / 4}\left|\log x-\log x_{n}\right| \leq \frac{2}{10^{n}} .
$$

Hence

$$
\begin{aligned}
x^{2} / T_{n} & \leq x^{2} \exp \left\{-(\log x)^{3 / 4}+2 / 10^{n}\right\} \\
& \leq x^{2} \exp \left\{-(\log x)^{3 / 4}+2 / 10\right\} \leq 2 x^{2} e^{-(\log x)^{3 / 4}} .
\end{aligned}
$$

From this and from equation (11) we get

$$
M(x)=k \varphi(1) \frac{1}{2} x^{2}+\sum_{m=-n}^{n} J_{m}+O\left(x^{2} e^{-(\log x)^{3 / 4}}\right),
$$

where the implied $O$-constant is less than $2 \cdot 192+|1-k| \varphi(0) \leq 384+3 \cdot 45=$ 519 . 
Now we show that all the terms in the sum (12) other than $J_{-n}$ and $J_{n}$ can be put into the error term.

Proposition 8. $\left|\sum_{n_{0}<|m| \leq n-1} J_{m}\right| \leq 120 x^{2} e^{-(\log x)^{3 / 4}}$.

Proof. It is easy to see that the contribution to the integral $J_{m}$ due to the loop circle centered at $1-a_{m}+i b_{m}$ tends to zero as the radius tends to zero. Hence we can write

$$
\begin{aligned}
\left|J_{m}\right| & =\left|\frac{1}{2 \pi i} \int_{C_{m}} \zeta(s) \frac{x^{s+1}}{s(s+1)} d s\right| \\
& \leq \frac{1}{\pi} \int_{-3 / 2}^{1-a_{m}} 45 \frac{x^{2-a_{m}}}{b_{m}^{2}} d \sigma \leq \frac{15}{b_{m}^{2}} x^{2} e^{-a_{m} \log x} .
\end{aligned}
$$

But if $|m| \leq n-1$ then

$$
\begin{aligned}
e^{-a_{m} \log x} & \leq e^{-a_{n-1} \log x} \\
& =\exp \left\{-\frac{\log x}{\left(\log x_{n}\right)^{1 / 4}}\right\} \leq \exp \left\{\frac{-\log x_{n}+2 / 10^{n}}{\left(\log x_{n}\right)^{1 / 4}}\right\} \\
& \leq \exp \left\{-\left(\log x_{n}\right)^{3 / 4}+2 / 10^{n}\right\} \leq \exp \left\{-(\log x)^{3 / 4}+4 / 10^{n}\right\} \\
& \leq \exp \left\{-(\log x)^{3 / 4}+4 / 10\right\} \leq 2 e^{-(\log x)^{3 / 4}} .
\end{aligned}
$$

Hence

$$
\left|\sum_{n_{0}<|m| \leq n-1} J_{m}\right| \leq 30 x^{2} e^{-(\log x)^{3 / 4}} \sum_{|m|>n_{0}} \frac{1}{b_{m}^{2}} .
$$

We finish the proof by noting that the last sum is finite:

$$
\sum_{|m|>n_{0}} \frac{1}{b_{m}^{2}} \leq \sum_{|m|>n_{0}} e^{-\sqrt{\log x_{m}}} \leq 2 \sum_{m>n_{0}} e^{-10^{m / 2}} \leq 2 \sum_{m>n_{0}} e^{-m} \leq 4 .
$$

Therefore we now see that

$$
M(x)=k \varphi(1) \frac{1}{2} x^{2}+\left(J_{-n}+J_{n}\right)+O\left(x^{2} e^{-(\log x)^{3 / 4}}\right),
$$

where the implied $O$-constant is less than $519+120=639$.

It remains to study the expression $J_{-n}+J_{n}$.

Denote by $J_{n}^{\prime}$ and $J_{n}^{\prime \prime}$ the integrals along the line segments $C_{n}^{\prime}$ and $C_{n}^{\prime \prime}$ lying respectively above and below the branch cut $C_{n}$ so that $J_{n}=J_{n}^{\prime}+J_{n}^{\prime \prime}$. Now, if we write

$$
s=1-a_{n}+i b_{n}+t e^{i \theta}, \quad-\pi \leq \theta<\pi,
$$

then the line segment $C_{n}^{\prime \prime}$ is obtained by letting $\theta=-\pi$ and $t$ run from 0 to 
$1-a_{n}+3 / 2$. In this way we obtain $C_{n}^{\prime \prime}$ with its direction reversed:

$$
-C_{n}^{\prime \prime}:\left\{\begin{array}{l}
\theta=-\pi, \\
s=1-a_{n}+i b_{n}-t, \\
d s=-d t \\
0 \leq t \leq 1-a_{n}+3 / 2 .
\end{array}\right.
$$

Hence

$$
\begin{aligned}
& =\frac{-1}{2 \pi i}\left\{\int_{0}^{(\log x)^{-1 / 4}}+\int_{(\log x)^{-1 / 4}}^{1-a_{n}+3 / 2}\right\} \frac{\zeta\left(1-a_{n}+i b_{n}-t\right) x^{2-a_{n}+i b_{n}-t}}{\left(1-a_{n}+i b_{n}-t\right)\left(2-a_{n}+i b_{n}-t\right)}(-d t) \\
& =\frac{1}{2 \pi i} \int_{0}^{(\log x)^{-1 / 4}} \frac{\zeta\left(1-a_{n}+i b_{n}-t\right) x^{2-a_{n}+i b_{n}-t}}{\left(1-a_{n}+i b_{n}-t\right)\left(2-a_{n}+i b_{n}-t\right)} d t+O\left(x^{2} e^{-(\log x)^{3 / 4}}\right),
\end{aligned}
$$

with the $O$-constant less than 1 . Indeed, since $b_{n}^{2} \geq 10$,

$$
\begin{aligned}
\left|\frac{1}{2 \pi i} \int_{(\log x)^{-1 / 4}}^{1-a_{n}+3 / 2} \ldots d t\right| & \leq \frac{1}{2 \pi} \cdot \frac{45}{b_{n}^{2}} x^{2-(\log x)^{-1 / 4}}\left(1-a_{n}+3 / 2-(\log x)^{-1 / 4}\right) \\
& \leq \frac{5}{4 \pi} \cdot \frac{45}{b_{n}^{2}} x^{2} \exp \left\{-\frac{\log x}{(\log x)^{1 / 4}}\right\} \leq x^{2} e^{-(\log x)^{3 / 4}} .
\end{aligned}
$$

Let us rewrite the integrand in expression (14):

$$
\begin{aligned}
\frac{\zeta(s)}{s(s+1)}= & \frac{s+k-1}{s(s-1)(s+1)} \prod_{|m|>n_{0}}\left(1-\frac{k}{s-1+a_{m}-i b_{m}+k}\right)^{\alpha_{m} / 2} \\
= & \left(1-\frac{k}{s-1+a_{n}-i b_{n}+k}\right)^{\alpha_{n} / 2} \frac{s+k-1}{s(s-1)(s+1)} \\
& \times \prod_{\substack{|m|>n_{0} \\
m \neq n}}\left(1-\frac{k}{s-1+a_{m}-i b_{m}+k}\right)^{\alpha_{m} / 2} \\
= & \left(s-1+a_{n}-i b_{n}\right)^{\alpha_{n} / 2} \\
& \quad \times \frac{(s+k-1) \prod_{\substack{|m|>n_{0} \\
m \neq n}}\left(1-\frac{k}{s-1+a_{m}-i b_{m}+k}\right)^{\alpha_{m} / 2}}{s(s-1)(s+1)\left(s-1+a_{n}-i b_{n}+k\right)^{\alpha_{n} / 2}} \\
= & \left(s-1+a_{n}-i b_{n}\right)^{\alpha_{n} / 2} f_{n}(s),
\end{aligned}
$$


where $f_{n}$ is an analytic function at $s=1-a_{n}+i b_{n}$ with a power series expansion having a radius of convergence greater than one:

$$
f_{n}(s)=\sum_{j=0}^{\infty} a_{n, j}\left(s-1+a_{n}-i b_{n}\right)^{j} .
$$

The integrand in (14) can now be written as

$$
\begin{aligned}
x^{2-a_{n}+i b_{n}-t}\left(t e^{-i \pi}\right)^{\alpha_{n} / 2} & f_{n}\left(1-a_{n}+i b_{n}-t\right) \\
& =x^{2-a_{n}+i b_{n}} \sum_{j=0}^{\infty} a_{n, j} x^{-t} t^{j+\alpha_{n} / 2} e^{-\pi i\left(j+\alpha_{n} / 2\right)} .
\end{aligned}
$$

Thus, writing $E$ in place of $O\left(x^{2} e^{-(\log x)^{3 / 4}}\right)$, we have

$$
\begin{aligned}
J_{n}^{\prime \prime}= & \frac{1}{2 \pi i} \int_{0}^{(\log x)^{-1 / 4}} x^{2-a_{n}+i b_{n}} \sum_{j=0}^{\infty} a_{n, j} x^{-t} t^{j+\alpha_{n} / 2} e^{-\pi i\left(j+\alpha_{n} / 2\right)} d t+E \\
= & \frac{1}{2 \pi i} x^{2-a_{n}+i b_{n}} \sum_{j=0}^{\infty} a_{n, j} e^{-\pi i\left(j+\alpha_{n} / 2\right)} \int_{0}^{(\log x)^{-1 / 4}} x^{-t} t^{j+\alpha_{n} / 2} d t+E \\
= & \frac{1}{2 \pi i} x^{2-a_{n}+i b_{n}} e^{-\pi i \alpha_{n} / 2} \sum_{j=0}^{\infty}(-1)^{j} a_{n, j} \int_{0}^{(\log x)^{-1 / 4}} x^{-t} t^{j+\alpha_{n} / 2} d t+E \\
= & \frac{1}{2 \pi i} x^{2-a_{n}+i b_{n}} e^{-\pi i \alpha_{n} / 2}\left(\frac{1}{\log x}\right)^{\alpha_{n} / 2+1} \\
& \times \sum_{j=0}^{\infty} a_{n, j}\left(\frac{-1}{\log x}\right)^{j(\log x)^{3 / 4}} \int_{0}^{-t} e^{j+\alpha_{n} / 2} d t+E \\
= & \frac{1}{2 \pi i} x^{2-a_{n}+i b_{n}} e^{-\pi i \alpha_{n} / 2}\left(\frac{1}{\log x}\right)^{\alpha_{n} / 2+1} \\
& \times \int_{0}^{(\log x)^{3 / 4}} e^{-t} t^{\alpha_{n} / 2} \sum_{j=0}^{\infty} a_{n, j}\left(\frac{-t}{\log x}\right)^{j} d t+E \\
= & \frac{1}{2 \pi i} x^{2-a_{n}+i b_{n}} e^{-\pi i \alpha_{n} / 2}\left(\frac{1}{\log x}\right)^{\alpha_{n} / 2+1} S_{n}+O\left(x^{2} e^{-(\log x)^{3 / 4}}\right)
\end{aligned}
$$

with

$$
S_{n}=\int_{0}^{(\log x)^{3 / 4}} e^{-t} t^{\alpha_{n} / 2} f_{n}\left(1-a_{n}+i b_{n}-\frac{t}{\log x}\right) d t .
$$


In a similar fashion we obtain

$$
J_{n}^{\prime}=\frac{-1}{2 \pi i} x^{2-a_{n}+i b_{n}} e^{\pi i \alpha_{n} / 2}\left(\frac{1}{\log x}\right)^{\alpha_{n} / 2+1} S_{n}+O\left(x^{2} e^{-(\log x)^{3 / 4}}\right) .
$$

We therefore have

(15)

$$
\begin{aligned}
J_{n} & =J_{n}^{\prime}+J_{n}^{\prime \prime} \\
& =-\frac{\sin \frac{\pi}{2} \alpha_{n}}{\pi} x^{2-a_{n}+i b_{n}}\left(\frac{1}{\log x}\right)^{\alpha_{n} / 2+1} S_{n}+O\left(x^{2} e^{-(\log x)^{3 / 4}}\right),
\end{aligned}
$$

with the $O$-constant not greater than 10 .

When we calculate $J_{-n}$ we obtain the complex conjugate of $J_{n}$ (this is because $b_{-n}=-b_{n}$ ). Therefore

$$
J_{n}+J_{-n}=J_{n}+\bar{J}_{n}=2 \Re\left(J_{n}\right) .
$$

Our next step is to estimate the integral $S_{n}$ appearing in (15). First we obtain lower and upper bounds for

$$
f_{n}(s)=\frac{s+3}{s(s-1)(s+1)} \cdot \frac{\prod_{\substack{|m|>n_{0} \\ m \neq n}}\left(1-\frac{k}{s-1+a_{m}-i b_{m}+k}\right)^{\alpha_{m} / 2}}{\left(s-1+a_{n}-i b_{n}+k\right)^{\alpha_{n} / 2}}
$$

when $\left|s-1+a_{n}-i b_{n}\right| \leq 1$.

For the upper bound we notice that

$$
|s|>b_{n}-1
$$

Thus

Also

$$
\left|\frac{s+3}{s(s-1)(s+1)}\right| \leq \frac{b_{n}+6}{\left(b_{n}-2\right)^{3}} \leq \frac{2 b_{n}}{\left(b_{n} / 2\right)^{3}}=\frac{16}{b_{n}^{2}} .
$$

$$
\left|s-1+a_{n}-i b_{n}+k\right|^{\alpha_{n} / 2}>\left(k-\left|s-1+a_{n}-i b_{n}\right|\right)^{\alpha_{n} / 2} \geq 3^{\alpha_{n} / 2} \geq 1 .
$$

Now we want to estimate from above the product appearing in the definition of $f_{n}$ (equation (16)). As in the proof of Proposition 5 we have

$$
\left|1-\frac{k}{s-1+a_{m}-i b_{m}+k}\right| \leq 1+\frac{k}{\left|\Im(s)-b_{m}\right|} \leq 1+\frac{k}{2 k}=\frac{3}{2} .
$$

Thus the product in (16) is less than

$$
\prod_{\substack{|m|>n_{0} \\ m \neq n}}\left(\frac{3}{2}\right)^{\alpha_{n} / 2} \leq \prod_{|m|>n_{0}}\left(\frac{3}{2}\right)^{1 / m^{2}} \leq\left(\frac{3}{2}\right)^{2 \sum_{j=1}^{\infty} 1 / j^{2}}<4 .
$$

Thus we have proved 
Proposition 9. For $\left|s-\left(1-a_{n}+i b_{n}\right)\right| \leq 1$,

$$
\left|f_{n}(s)\right| \leq 64 / b_{n}^{2} \text {. }
$$

This and Cauchy's inequalities give the following

Corollary 10. For all $j=1,2, \ldots$,

$$
\left|a_{n, j}\right| \leq 64 / b_{n}^{2}
$$

Now for the lower bound:

$|s| \leq\left|s-1+a_{n}-i b_{n}\right|+\left|1-a_{n}+i b_{n}\right| \leq 1+1+\left|a_{n}\right|+\left|b_{n}\right| \leq 3+b_{n}$.

Thus

$\left|\frac{s+3}{s(s-1)(s+1)}\right| \geq \frac{|s+3|}{\left(b_{n}+4\right)^{3}} \geq \frac{|s|-3}{\left(b_{n}+4\right)^{3}} \geq \frac{b_{n}-1-3}{\left(b_{n}+4\right)^{3}} \geq \frac{\frac{1}{2} b_{n}}{\left(2 b_{n}\right)^{3}}=\frac{1}{16 b_{n}^{2}}$.

Each term in the infinite product in (16) is

$$
\left|1-\frac{k}{s-1+a_{m}-i b_{m}+k}\right| \geq 1-\frac{k}{\left|s-1+a_{m}-i b_{m}+k\right|} \geq 1-\frac{k}{2 k}=\frac{1}{2} \text {. }
$$

Therefore

$$
\begin{aligned}
& \prod_{\substack{|m|>n_{0} \\
m \neq n}}\left(1-\frac{k}{s-1+a_{m}-i b_{m}+k}\right)^{\alpha_{n} / 2} \\
& \quad \geq \prod_{|m|>0}\left(\frac{1}{2}\right)^{1 / m^{2}}=\left(\frac{1}{2}\right)^{2 \sum_{m=1}^{\infty} 1 / m^{2}}=\left(\frac{1}{2}\right)^{\pi^{2} / 3}>\frac{1}{10} .
\end{aligned}
$$

Thus we have

Proposition 11. For $\left|s-\left(1-a_{n}+i b_{n}\right)\right| \leq 1$ we have

$$
\left|f_{n}(s)\right| \geq \frac{1}{16 b_{n}^{2}} \cdot \frac{1}{5} \cdot \frac{1}{10}=\frac{1}{800 b_{n}^{2}} .
$$

With all these inequalities we can estimate the integral for $S_{n}$, the function occurring in (15), as follows:

$$
\begin{aligned}
S_{n} & =\int_{0}^{(\log x)^{3 / 4}} e^{-t} t^{\alpha_{n} / 2} f_{n}\left(1-a_{n}+i b_{n}-\frac{t}{\log x}\right) d t \\
& =\int_{0}^{(\log x)^{3 / 4}} e^{-t} t^{\alpha_{n} / 2} \sum_{j=0}^{\infty} a_{n, j}\left(\frac{-t}{\log x}\right)^{j} d t \\
& =a_{n, 0} \int_{0}^{(\log x)^{3 / 4}} e^{-t} t^{\alpha_{n} / 2} d t+\sum_{j=1}^{\infty} a_{n, j} \int_{0}^{(\log x)^{3 / 4}} e^{-t} t^{\alpha_{n} / 2}\left(\frac{-t}{\log x}\right)^{j} d t .
\end{aligned}
$$


For the second term we get, by Corollary 10,

$$
\begin{aligned}
\left|\sum_{j=1}^{\infty} a_{n, j} \int_{0}^{(\log x)^{3 / 4}} e^{-t} t^{\alpha_{n} / 2}\left(\frac{-t}{\log x}\right)^{j} d t\right| & \leq \sum_{j=1}^{\infty} \frac{64}{b_{n}^{2}}\left(\frac{1}{\log x}\right)^{j / 4} \int_{0}^{\infty} e^{-t} t^{\alpha_{n} / 2} d t \\
& \leq\left(\frac{1}{\log x}\right)^{1 / 4} \frac{64}{b_{n}^{2}} \sum_{j=0}^{\infty}\left(\frac{10}{98}\right)^{j / 4} \\
& \leq 148\left(\frac{1}{\log x}\right)^{1 / 4} \frac{1}{b_{n}^{2}} .
\end{aligned}
$$

Now, since

$$
\int_{(\log x)^{3 / 4}}^{\infty} e^{-t} t^{\alpha_{n} / 2} d t \leq \int_{(\log x)^{3 / 4}}^{\infty} e^{-t} t d t \leq 2 \log x e^{-(\log x)^{3 / 4}}
$$

the integral $S_{n}$ in (15) is

$$
S_{n}=a_{n, 0}\left(\Gamma\left(\frac{1}{2} \alpha_{n}+1\right)+\theta_{2} \log x e^{-(\log x)^{3 / 4}}\right)+\frac{\theta_{3}}{b_{n}^{2}(\log x)^{1 / 4}},
$$

where $\left|\theta_{2}\right| \leq 2$ and $\left|\theta_{3}\right| \leq 148$. Since $a_{n, 0}=f_{n}\left(1-a_{n}+i b_{n}\right)$, from Proposition 11 and from $\Gamma\left(\frac{1}{2} \alpha_{n}+1\right)>0.8$ we find that the modulus of $S_{n}$ is greater than

$$
\begin{aligned}
& \frac{1}{800 b_{n}^{2}}\left(0.8-2 \log x e^{-(\log x)^{3 / 4}}\right)-\frac{148}{b_{n}^{2}(\log x)^{1 / 4}} \\
& =\frac{1}{800 b_{n}^{2}}\left(0.8-2 \log x e^{-(\log x)^{3 / 4}}-\frac{118400}{(\log x)^{1 / 4}}\right) \geq \frac{e^{-2\left(\log x_{n}\right)^{1 / 2}}}{1600}
\end{aligned}
$$

for $x \geq X_{1}$, i.e., if $x$ is sufficiently large.

We will use this lower bound for the integral $S_{n}$ appearing in equation (15). Now consider the other factor in that equation,

$$
\begin{aligned}
\frac{\sin \frac{\pi}{2} \alpha_{n}}{\pi} x^{2-a_{n}}\left(\frac{1}{\log x}\right)^{\alpha_{n} / 2+1} & \geq \frac{\alpha_{n}}{\pi} x^{2} e^{-\left(\log x_{n}\right)^{1 / 2}} \frac{1}{2(\log x)^{2}} \\
& \geq \frac{x^{2}}{\pi} e^{-\left(\log x_{n}\right)^{1 / 2}} \frac{1}{2(\log x)^{2}\left(\log \log x_{n}\right)^{2}} .
\end{aligned}
$$

From the above and equation (15),

$$
\left|J_{n}\right| \geq \frac{x^{2}}{\pi 1600} \cdot \frac{e^{-3\left(\log x_{n}\right)^{1 / 2}}}{4(\log x)^{4}} \geq \frac{10^{-5}}{(\log x)^{4}} e^{-3(\log x)^{1 / 2}} \quad \text { if } x \geq X_{1} .
$$

We would like $J_{n}+J_{-n}=2 \Re\left(J_{n}\right)$ to be large. We already know that $\left|J_{n}\right|$ is large, but still it can be that $\Re\left(J_{n}\right)=0$, say. Let us recall here equation 
(15):

$$
J_{n}=-\frac{\sin \frac{\pi}{2} \alpha_{n}}{\pi} x^{2-a_{n}+i b_{n}}\left(\frac{1}{\log x}\right)^{\alpha_{n} / 2+1} S_{n}+O\left(x^{2} e^{-(\log x)^{3 / 4}}\right),
$$

where $x=x_{n}\left(1+\theta_{1} / \log x_{n}\right),\left|\theta_{1}\right|<1$. Now express this equation as

$$
A:=\frac{J_{n}}{x^{2-a_{n}}}=B x^{i b_{n}}+C,
$$

where $B$ and $C$ have obvious definitions. Dividing by $|B|$ we get

$$
\begin{aligned}
\Re\left(\frac{A-C}{|B|}\right) & =\Re\left(\exp \left\{i b_{n} \log x+i \arg B\right\}\right)=\cos \left(b_{n} \log x+\arg B\right) \\
& =\cos \left(b_{n}\left(\log x_{n}+\log \left(1+\frac{\theta_{1}}{\log x_{n}}\right)\right)+\arg B\right) \\
& =\cos \left(\left\{b_{n} \log x_{n}+\arg B\right\}+b_{n} \log \left(1+\frac{\theta_{1}}{\log x_{n}}\right)\right) .
\end{aligned}
$$

Notice that $\arg B$ is a function of $\theta_{1}$. This is because $x$ is a function of $\theta_{1}$ and $S_{n}$ is a function of $x$. Hence we have

$$
\arg B=\arg S_{n}+\pi \text {. }
$$

The main term on the right hand side of (17) is independent of $\theta_{1}$. Since the other two terms are much smaller, we see that as $\theta_{1}$ runs from -1 to +1 , the argument of $S_{n}$ (and hence of $B$ ) undergoes a change not greater than $2 \pi$.

Therefore, as $\theta_{1}$ runs from -1 to +1 , the argument of the above cosine runs through an interval centered somewhere in

$$
\left(b_{n} \log x_{n}-2 \pi, b_{n} \log x_{n}+2 \pi\right)
$$

and having a length greater than

$$
\begin{aligned}
b_{n} \log \left(1+\frac{1}{\log x_{n}}\right) & =b_{n} \int_{1}^{1+\left(\log x_{n}\right)^{-1}} \frac{d t}{t} \geq b_{n}\left(\frac{1}{1+\left(\log x_{n}\right)^{-1}}\right)\left(\frac{1}{\log x_{n}}\right) \\
& \geq b_{n} \frac{10}{11} \cdot \frac{1}{\log x_{n}}=\frac{10}{11} \cdot \frac{e^{\sqrt{\log x_{n}}}}{\log x_{n}} \rightarrow \infty \quad \text { as } n \rightarrow \infty .
\end{aligned}
$$

Therefore, when $n$ is large we can choose values, $\theta_{1}(+)$ and $\theta_{1}(-)$, of $\theta_{1}$ such that

$$
\Re\left(\frac{A-C}{|B|}\right)=+1 \quad \text { and } \quad \Re\left(\frac{A-C}{|B|}\right)=-1 .
$$

In the first case we get

$$
\Re\left(\frac{J_{n}}{x^{2-a_{n}}}\right)=\Re(A)=|B|+\Re(C),
$$


or, what is the same,

$$
\begin{aligned}
\Re\left(J_{n}\right) & =|B| x^{2-a_{n}}+\Re(C) x^{2-a_{n}} \\
& \geq\left|J_{n}\right|-|C| x^{2-a_{n}}+\Re(C) x^{2-a_{n}} \\
& =\left|J_{n}\right|+O\left(x^{2} e^{-(\log x)^{3 / 4}}\right) \\
& \geq \frac{10^{-5}}{2(\log x)^{4}} e^{-3(\log x)^{1 / 2}} \quad \text { if } x \geq X_{1} \text { and } \theta_{1}=\theta_{1}(+) .
\end{aligned}
$$

In a similar fashion we obtain

$$
\Re\left(J_{n}\right) \leq-\frac{10^{-5}}{2(\log x)^{4}} e^{-3(\log x)^{1 / 2}} \quad \text { if } x \geq X_{1} \text { and } \theta_{1}=\theta_{1}(-) .
$$

Notice that the inequalities (19) and (20) hold when

$$
x \geq X_{1}, \quad x=x_{n}\left(1+\frac{\theta_{1}}{\log x_{n}}\right), \quad x_{n}=\exp \left\{10^{2^{n-1}}\right\}, \quad \theta_{1}=\theta_{1}( \pm) .
$$

These inequalities and the equation

$$
M(x)=2 \varphi(1) x^{2}+2 \Re\left(J_{n}\right)+O\left(x^{2} e^{-(\log x)^{3 / 4}}\right)
$$

imply relation (8); in fact we have proved the following stronger statement:

$$
M(x)=2 \varphi(1) x^{2}+\Omega_{ \pm}\left(x^{2} e^{-(3+\varepsilon)(\log x)^{1 / 2}}\right) \quad \forall \varepsilon>0 .
$$

\section{References}

[1] A. Beurling, Analyse de la loi asymptotique de la distribution des nombres premiers généralisés. I, Acta Math. 68 (1937), 255-291.

[2] H. G. Diamond, The Prime Number Theorem for Beurling's generalized numbers, J. Number Theory 1 (1969), 200-207.

[3] - , Asymptotic distribution of Beurling's generalized integers, Illinois J. Math. 14 (1970), 12-28.

[4] P. Malliavin, Sur le reste de la loi asymptotique de répartition des nombres premiers généralisés de Beurling, Acta Math. 106 (1961), 281-298.

Instituto de Matemáticas

UNAM-Morelia

Nicolás Romero 150 Centro

58000 Morelia, Michoacán

México

E-mail: balanzar@math.uiuc.edu balanz@jupiter.ccu.umich.mx 